\title{
CORRESPONDENCE
}

\section{Long-term neuroprotection of retinal ganglion cells by inhibiting caspase-2}

Cell Death Discovery (2016) 2, 16044; doi:10.1038/cddiscovery.2016.44; published online 13 June 2016

Caspases are a family of cysteine-rich proteases that activate apoptotic pathways through their 'initiator' or 'executioner' pathways. Initiator caspases activate executioner caspase, such as caspase- $2,-3,-6$ and -7 , which hydrolyze or cleave structural proteins in the cell leading to apoptosis. Caspase-2 is the most highly conserved caspase and uniquely fits into both initiator and executioner categories and as such partakes in a variety of apoptosis inducing signals including DNA damage, heat shock, endoplasmic reticulum and oxidative stress. Caspase-2-deficient neurons are resistant to apoptosis induced by beta-amyloid although it is involved in apoptosis of hippocampal neurons after ischemia and is expressed in ischemic retinae. ${ }^{1}$

The protection of retinal ganglion cells (RGC), particularly after trauma and numerous ocular pathologies such as glaucoma and ischemic optic neuropathy, is of particular interest to the ocular biology field. RGC loss, a hallmark of these diseases leads to vision loss and blindness. ${ }^{2}$ RGC are particularly susceptible and in the rat optic nerve transection/crush model in adult rats, RGC die rapidly and within 28 days, $90 \%$ of RGC are lost. ${ }^{3}$ As apoptosis accounts for the majority of RGC death, treatments that inhibit apoptosis afford limited neuroprotection, including microglial inhibitors, brain-derived neurotrophic factor, overexpression of $\mathrm{BCl}-2$ and caspase inhibitors. ${ }^{4}$ Caspase- $3,-6,-8$ and -9 are all regarded as important for RGC apoptosis, however, inhibition of these caspases, either alone or in specific combinations afforded protection of up to $60 \%$ of RGC. ${ }^{5}$ Furthermore, our observations that cleaved caspase- 3 was absent in RGC and that little or no changes in cleaved caspase- 6 and -7 were detected after transection of RGC axons by optic nerve crush (ONC) suggested that other caspases may have a part in RGC apoptosis. ${ }^{3,6}$

We previously reported that RGCs exclusively expressed activated (cleaved) caspase-2 after ONC in adult rats and confirmed these findings by employing an unbiased caspase trapping assay. 3 . We further showed that inhibition of caspase-2 by either a pharmacological inhibitor ${ }^{6}$ or a chemically modified synthetic short interfering RNA (siCASP2) significantly protected RGC from death, the latter protecting $>95 \%$ of RGC from death for up to 21 days after ONC. ${ }^{3,7}$ Here, we demonstrate in retinal whole mounts, prepared after FluroGold backfilling of $\mathrm{RGC}_{1}^{3,7}$ that intravitreal injection of siCASP2 every 8 days protected RGC from death at all radial quadrants from the optic disc including the central, mid-periphery and outer quadrants, with $>95 \%$ protection of RGC from death over the entire retina at 12 weeks after ONC (Supplementary Figure S1), compared with intact controls $\left(2170 \pm 200\right.$ in siCASP2 treated eyes versus $2250 \pm 230 \mathrm{RGC} / \mathrm{mm}^{2}$ in intact controls). Only a small proportion of RGC were present in retinal whole mounts from control scrambled siRNA-(siCNL $\left.{ }^{3}\right)$ treated eyes $\left(150 \pm 130 \mathrm{RGC} / \mathrm{mm}^{2}\right)$. Our results suggest that neuroprotection by siCASP2 is extremely efficient throughout the entire retina and that this is an unprecedented level of neuroprotection. To the best of our knowledge, our results far exceed those of any others published in the literature. Our results affirm that caspase- 2 is the main caspase responsible for the death of RGC after direct trauma. This bodes well for siCASP2, otherwise known as QPI-1007, currently recruiting patients in a worldwide Phase III trial that is being initiated by Quark Pharmaceuticals Inc (USA) (http://quarkpharma.com/?p = 12241).

\section{COMPETING INTERESTS}

The authors declare no conflict of interest.

\section{ACKNOWLEDGEMENTS}

We thank Quark Pharmaceuticals Inc for providing their proprietary siRNA, QPI-1007, targeting caspase-2 (siCASP2) and the control scrambled siRNA (siCNL). This work was funded by a Wellcome Trust project grant no. 092539/Z/10/Z to ZA.

V Vigneswara and Z Ahmed Neurotrauma Research Group, Institute of Inflammation and Ageing, College of Medical and Dental Sciences, University of Birmingham, Birmingham, UK Correspondence: Z Ahmed (z.ahmed.1@bham.ac.uk)

\section{REFERENCES}

1 Troy CM et al. J Neurosci 2000; 20: 1386-1392.

2 Kisiswa L et al. Exp Eye Res 2010; 91: 739-747.

3 Ahmed Z et al. Cell Death Dis 2011; 2: e173.

4 Chierzi $S$ et al. Vision Res 1998; 38: 1537-1543.

5 Monnier PP et al. J Neurosci 2011; 31: 10494-10505.

6 Vigneswara $V$ et al. PLoS One 2012; 7: e53473.

7 Vigneswara $V$ et al. Brain 2014; 137: 1656-1675.

This work is licensed under a Creative Commons Attribution 4.0 International License. The images or other third party material in this article are included in the article's Creative Commons license, unless indicated otherwise in the credit line; if the material is not included under the Creative Commons license, users will need to obtain permission from the license holder to reproduce the material. To view a copy of this license, visit http://creativecommons.org/licenses/ by/4.0/

(c) The Author(s) 2016

Supplementary Information accompanies the paper on the Cell Death and Discovery website (http://www.nature.com/cddiscovery) 